\title{
Dietary intake of vitamin $D$ in adults with overweight and obesity
}

\author{
Maria Nikolova and Alexander Penkov \\ Medical University - Sofia; Department of Hygiene, Medical Ecology and Nutrition, Sofia, Bulgaria
}

\begin{abstract}
Introduction: The aim of the present study is to study in detail the dietary intake of vitamin D in subjects with different BMI in relation to the status of serum vitamin D.
\end{abstract}

Material and Methods: They have been studied 264 individuals (41.3\% were men and 58.9\% were women), aged 19 to 60 years. Body weight and height, waist circumference, systolic and diastolic blood pressure were recorded. Body composition was assessed by bioelectrical impedance. Serum 25(OH)D Total, insulin,high-sensitivity C-reactive protein, glucose level and lipids were measured. Nutrition evaluation was performed through a 24-hour recall and FFQ in January-April 2014 and 2015.

Results: $27.2 \%$ of the participants had normal weight, $24.6 \%$ - overweight, $29.2 \%$ - class I obesity, and $18.9 \%$ - class II or III. $33.3 \%$ had vitamin D deficiency, $40.2 \%$ - insufficiency. It was found that the average daily intake of vitamin D for the whole group of subjects was $6.6 \mathrm{mcg} / \mathrm{day}$. (for the women $-5.6 \mu \mathrm{g} / \mathrm{d}$ and for the men $-10.4 \mu \mathrm{g} / \mathrm{d}$ ). In BMI-defined groups it was found that daily average daily intakes of vitamin D in subjects with normal BMI were $7.6 \mathrm{mcg} / \mathrm{day}$, in those with overweight was $6.6 \mathrm{mcg} / \mathrm{d}$, and in those with obesity was $6.0 \mathrm{mcg} /$ day. $80.6 \%$ of the subjects were found to have daily average daily intakes of vitamin D under the EAR, which determines a high relative share of individuals with a potential risk of vitamin D deficiency. We found significant, mild to moderate correlations between daily average dietary intake of vitamin D and weight, \% body fat (\% BF); fat mass (FM), visceral fat (in women), muscle and fat-free mass (FFM), and height-adjusted indices : fat mass index (FMI) and fat-free mass index (FFMI), total body water (TBW in kg and \%) and with some of metabolic variables (basal insulin and with the chronic inflammation marker - hs-CRP.

Discussion: Dietary intake of vitamin D is insufficient and correlates with serum vitamin D levels, FM(kg), \%FM, visceral fat, FFM, muscle mass and some of metabolic variables. Male sex, higher education, younger than 30 years and normal BMI (up to $25.0 \mathrm{~kg} / \mathrm{m} 2$ ) are the factors determining the higher dietary intake of vitamin D. This requires targeted supplementation and additional intake of vitamin $\mathrm{D}$ fortified foods to individuals at risk.

\section{Conflict of Interest}

There is no conflict of interest 